\title{
Cost-Efficient Deployment of Multi-hop Wireless Networks over Disaster Areas using Multi-Objective Meta-Heuristics
}

\author{
M. N. Bilbao ${ }^{\text {a }}$, J. Del Ser ${ }^{a, b, *}$, C. Perfecto ${ }^{a}$, S. Salcedo-Sanz ${ }^{c}$ \\ and J. A. Portilla-Figueras ${ }^{\mathrm{c}}$ \\ ${ }^{a}$ University of the Basque Country UPV/EHU, 48013 Bilbao, Spain. \\ b OPTIMA Area, TECNALIA, 48160 Derio, Bizkaia, Spain. \\ ${ }^{\mathrm{c}}$ Universidad de Alcalá, 28871 Alcalá de Henares, Madrid, Spain.
}

\begin{abstract}
Nowadays there is a global concern with the growing frequency and magnitude of natural disasters, many of them associated with climate change at a global scale. When tackled during a stringent economic era, the allocation of resources to efficiently deal with such disaster situations (e.g. brigades, vehicles and other support equipment for fire events) undergoes severe budgetary limitations which, in several proven cases, have lead to personal casualties due to a reduced support equipment. As such, the lack of enough communication resources to cover the disaster area at hand may cause a risky radio isolation of the deployed teams and ultimately fatal implications, as occurred in different recent episodes in Spain and USA during the last decade. This issue becomes even more dramatic when understood jointly with the strong budget cuts lately imposed by national authorities. In this context, this article postulates cost-efficient multi-hop communications as a technological solution to provide extended radio coverage to the deployed teams over disaster areas. Specifically, a Harmony Search (HS) based scheme is proposed to determine the optimal number, position and model of a set of wireless relays that must be deployed over a large-scale disaster area. The approach presented in this paper operates under a Pareto-optimal strategy, so a number of different deployments is then produced by balancing between redundant coverage and economical cost of the deployment. This information can assist authorities in their resource provisioning and/or operation duties. The performance of different heuristic operators to enhance the proposed HS algorithm are assessed and discussed by means of extensive simulations over synthetically generated scenarios, as well as over a more realistic, orography-aware setup constructed with LIDAR (Laser Imaging Detection and Ranging) data captured in the city center of Bilbao (Spain).
\end{abstract}

Key words: Disaster Communications; Multi-hop Relaying; Multi-objective Optimization; Genetic Algorithm; Harmony Search 


\section{Introduction}

In the last few years different studies based on observed evidences or databased experiments have shown that the number, frequency and severity of disaster events are sharply increasing around the world [1]. The main rationale for this upsurge of earthquakes, hurricanes, tropical storms, large-area wildfires and other events alike mainly lies in the widely-debated global warming phenomena, which has undoubtedly modified the natural environment of wide geographic areas (e.g. soil dryness, vegetation, orography and other related factors) and increased the risk exposure of the population [2]. Such abrupt changes impact on analytical indicators and climate models, which mostly rely on the relative stationarity of the climate in the short-medium term. Consequently, the effect of global warming on the occurrence of natural disasters has been so far assessed under a several-years-long perspective [3,4].

One of the large-area disasters undergoing a clear increase in its scales, magnitudes and consequences are wildfires. In this context, the report presented at the annual meeting of the American Geophysical Union in San Francisco (USA) in late 2012 predicted that the burned area from wildfires in the USA would double in size by 2050 due to warmer and drier conditions in forthcoming decades [6]. This prediction was buttressed by the record of incidences in that same year, with massive fires affecting Colorado and New Mexico [7]. In addition, when focusing on the spatio-temporal correlation of these events fire events have recurrently happened in nearby locations and close in time due to the propagative essence of the fire when held in isolated areas. A conclusion of utmost importance for the scope of this work is that all the above observations lead to the certainty that commanders and decision makers will encounter higher difficulties in the future when allocating resources against disasters due to their simultaneity, co-locality and interconnection.

Decisions in this context are driven by well-specified procedures and protocols based on the passive reaction triggered by circumstantial conditions (e.g. a given number of resources for every hectare of terrain affected by the disaster). For instance, several international protocols related to disaster risk reduction have been lately under development by different countries at a worldwide scale, such as the Hyogo Framework for Action (HFA) 2005-2015 [8], the SENDAI Framework for disaster risk reduction 2015-2030 [9,10], the ASEAN Agreement on Disaster Management and Emergency Response (AADMER) [11] and other similar national initiatives. However, cases such as the wildfire happened in a brush-choked canyon north of Phoenix (Arizona, USA) in

\footnotetext{
* Corresponding author: Dr. Javier Del Ser. TECNALIA RESEARCH \& INNOVATION, P. Tecnologico, Ed. 700, 48160 Derio, Bizkaia, Spain. T: +34 664113013. Fax: n.d.
}

Email address: javier.delser@tecnalia.com (J. Del Ser). 
June 2013 and studies reported in the literature $[12,13]$ showed up the fact that human decision making is subject to errors, partial information and assumptions that may lead to fatalities: in the aforementioned wildfire 19 elite firefighters perished while commanders thought the crew was in a safe place [14]. No extreme had heard each other for 33 minutes until just before the fire overwhelmed the brigade. In fact there is a plethora of examples where the fatal consequences of team isolation and lack of coordination in wildfires, such as the catastrophic wildfire happened in Guadalajara (Spain) in 2005 [15], or the one occurred in Lüneburg Heath (Lower Saxony, Germany) in 1975 [16]. Certainly decision support tools would have been extremely useful to deploy communication resources in a more effective, active, monitored fashion, discarding any non-supported assumptions from the commanding forces.

When approached from a budgetary perspective, safety should be a reason enough to allocate as much financial resources as available, so as to minimize risk likelihood and consequences severity. Nonetheless, the worldwide economical context of the last few years has restricted stringently national budget items allocated to fire prevention strategies and disaster management methodologies by institutions and governments [17]. For instance, the environmental forum of Castilla La Mancha region (center of Spain) denounces, in their report published in October 2013 [18], that there are only 5 light vehicles in the region with the legally required equipment to combat wildfires (one per province), which incur in delays and an increased risk when performing their duty. Besides, also in that report it is claimed that regional firefighting brigades have undergone significant reductions - reaching up to $50 \%$ - in the number of effective hours dedicated to the prevention, surveillance and extinction of wildfires. Even more exemplifying is the fact that the Spanish firefighting campaign for 2013 has dedicated 267 aircrafts for the extinction of wildfires during summertime, 8 units less than in 2012 as a consequence of the funding reductions in the Ministry for Agriculture, Fisheries and Food. Cost, therefore, is called to play an essential role when allocating resources nowadays and in the present future. Current resource allocation procedures, on the contrary, do not take into account any cost criteria in decision making.

Several references from the related literature have addressed this lack of cost effectiveness in the management of resources in disaster situations. For instance, the work in [19] analyzed bidding mechanisms for optimally procuring goods in disaster relief logistics by using an integer programming formulation of the underlying auction operation. The authors in [20] derived a dynamic time-dependent nonlinear model to quantify the influence of a disaster on supply, demand, and humanitarian logistics, and applied Genetic Algorithms to infer the optimal logistic plan minimizing mismatches between the supplied goods and the demand, as well as the time delay of their delivery. Other references in disaster management include the allocation of critical emergency resources in multi-hazard situations [21-23], transport and provisioning under 
uncertainty [24], supply chain logistics [25] and waste management [26], among many others.

In this context this paper elaborates on a particular yet usual resource allocation problem in disaster management: the deployment of wireless communication relays over a large-scale area wildfire. In practice, the heterogeneity of relay equipment is specially acute in terms of their coverage and cost, ranging from traditional $\mathrm{RF}$ front-ends installed on ground vehicles and portable masts to last-generation devices operating from unmanned aerial fleets of drones, helicopters and airplanes. This diversity in the set of deployable relays has led to a large amount of research, gravitating on the similarity of this paradigm with the well-known disk covering problem [27] when exclusively driven by coverage criteria (see e.g. [28-31] and references therein). However, it was not until recently [33] when the authors proposed to include cost aspects in the deployment of relays, as well as in predictive resource provisioning [32]. Following this discussion, this paper builds upon previous work by extending the problem in [33] with the possibility to establish multi-hop links between outof-coverage brigade units and the relays themselves through other brigades deployed in-between. To efficiently tackle the resulting bi-objective problem, a meta-heuristic solver inspired from music composition is proposed. This manuscript extends early work by the authors in [34] by including the following novel ingredients:

- A thorough survey of the state of the art in what relates to meta-heuristic solvers for communications in disaster scenarios.

- A variant of the problem formulated in [34] aimed at maximizing not only the cost efficiency and coverage of the relay deployment, but also at providing redundancy in the communications of the deployed teams upon eventual cuts in the radio links.

- A modification of the meta-heuristic algorithm aimed at jointly estimating the number of relays to be deployed in addition to their location and model.

- A comparison of the multi-objective performance of the proposed HS-based algorithm with respect to Genetic Algorithms (GA) - which are among the most utilized meta-heuristic solvers in the related literature, as will be later argued - with the same solution encoding scheme.

- Additional simulations over a more realistic problem setup over the city of Bilbao (Bizkaia, Spain) using open LIDAR (Laser Imaging Detection and Ranging) data to estimate realistic line-of-sight coverage areas. As will be shown in the article, the obtained results evince the good performance of the proposed scheme under different values of the admissible number of hops, and pave the way towards its application in real disaster situations.

The rest of the paper is structured as follows: Section 2 surveys the literature related to the technical scope of this article, and Section 3 mathematically formulates the considered problem. Next, Section 4 describes the proposed 
meta-heuristic solver, whereas Section 5 discusses the obtained simulation results. Finally, Section 6 ends the paper by drawing some concluding remarks.

\section{Related Work}

The growing use of mobile devices during the last decade has allowed for a better and quicker crisis response against disaster events [35]. In such situations, the need for an efficient deployment of wireless communication relays is an extremely challenging real problem to be tackled. It requires interoperability among highly heterogeneous relay equipment in terms of coverage, cost, autonomy and other similar factors, ranging from traditional RF front-ends installed on ground vehicles and portable masts to last-generation devices operating from unmanned aerial fleets of drones, helicopters and airplanes. The LTE (Long Term Evolution) cellular communications standard has been widely referred to as the key to efficiently unleash future public safety communications [36]. However, until this technology is completely deployed the diversity in the set of deployable relays has produced a lot of research directly related to disaster/emergency response communications deployment (recently surveyed by [37]), or indirectly related in the form of akin mobile ad-hoc networking, wireless mesh (WMNs) or wireless sensor networks (WSNs). Relay/node deployment represents a very active research niche in WMNs as pointed out in [38-42] and more recently in [43].

To proceed with the literature review about solvers for the optimum node deployment problem in a technology-agnostic manner, we will extend our study to WSNs under the assumption that their critically resource-constrained nodes do not constitute but one of the vast heterogeneity of possible relay technologies for disaster situations. Up to twenty recent contributions are summarized and analyzed in [44] focusing on the application of bio-inspired algorithms - Particle Swarm (PSO), Ant Colony Optimization (ACO) and Genetic Algorithms (GA) - to WSN resource allocation problems. In this study it is concluded that the centralized nature of PSO makes it the best candidate to minimize coverage holes when dealing with stationary node positioning. On the contrary, the distributed nature of ACO is better to solve mobile node deployment. Similarly, the work in [45] addresses the gateway placement problem using a nature inspired meta-heuristic algorithm coupled with a clustering approach. In [46] PSO, ACO and GA are compared to each other when dealing with the optimal placement of gateway nodes in WMNs. A multi-objective GA was utilized in [47] to balance between installation cost and coverage probability, and more recently [48] resorted to a variety of GAs, trajectory-based algorithms and two novel swarm intelligence solvers to address a three-objective optimization problem for relay node deployment in WSNs. Simulated Annealing (SA) alone or hybridized with GA has also been successfully applied to WMN deployment [49-51]. It is worth mentioning approaches related to 
multi-hop communication deployment in emergency/disaster scenarios such as [52], which used a distributed bio-inspired algorithm to select the role of each stem node in the network, or [53], which proposed a 802.11 system based on self-aligning directional antennas.

Finally, from the algorithmic perspective several authors have tackled different optimization problems related to wireless devices by means of other bioinspired solvers. In addition to those surveyed above in the context of WMNs (resorting mainly to GA and Swarm Intelligence), several new meta-heuristic algorithms are grasping the attention of the community working on the design of wireless communication networks. In this particular application field, one of the most actively explored bio-inspired solvers in the last years is the so-called Firefly algorithm [54], which hinges on the mutual attractiveness of these insects based on their brightness and its combination with typical operators from Swarm Intelligence to yield a bio-inspired optimizer already used for routing [55], node clustering [56] and localization [57]. Similarly, Cuckoo Search [58] imitates the particular behavior of this family of birds to efficiently cope with optimization problems with the additional advantage of being tunable by just one single parameter in its original formulation. This solver has been utilized for problems stemming from wireless networks, e.g. data aggregation [59], localization [60] or cluster formation [61]. Results have been also reported using other nature-inspired techniques farther from the literature mainstream, such as the so-called Social Emotional Optimization Algorithm [62], Intelligent Water Drops [63], Bat Algorithm [64], Bacterial Foraging [65] and Inmune Systems [66], among others.

Notwithstanding the flurry of research around nature-inspired optimization (and in general, bio-inspired processing) for wireless network applications, it is a common belief $[69,70]$ that as many techniques as possible should be explored towards overcoming the large gap from the simulation of this kind of methods to their implementation in practical systems. This observation is indeed where the contribution of our research work is framed: to the best of our knowledge the deployment of multi-hop wireless networks under a multiobjective cost-coverage criterion and redundancy constraints has not been approached yet using HS-based heuristics nor tested in realistic simulation setups, hence advancing over recent contributions using this algorithm that focus instead on maximizing the energy efficiency of fixed networks $[67,68]$.

\section{System Model and Problem Statement}

In reference to Figure 1, we assume $N$ teams operating over an area $A \subset \mathbb{R}^{2}$ affected by a disaster event. We let $\left\{\mathbf{c}_{n}\right\}_{n=1}^{N} \triangleq\left\{\left(x_{n}, y_{n}\right)\right\}_{n=1}^{N}$ and $\left\{r_{n}\right\}_{n=1}^{N}$ define their respective locations and coverage radii. In this scenario, $M$ relay devices 
are to be deployed at coordinates $\left\{\mathbf{c}_{m}^{R}\right\}_{m=1}^{M} \triangleq\left\{\left(x_{m}^{R}, y_{m}^{R}\right)\right\}_{m=1}^{M}$, each belonging to a class within the set $\left\{\Phi_{t}\right\}_{t=1}^{\tau}=\left\{\left(R_{t}, \beta_{t}\right)\right\}_{t=1}^{\tau}$, which represents the $\tau$ available relay models with radii $R_{t}$ and cost $\beta_{t}$ (in monetary units or m.u.). Intuitively yet realistically the model imposes that the larger the coverage radius $R_{t}$ is, the higher $\beta_{t}$ will be. A general (not necessarily injective nor surjective) mapping function $\phi:\{1, \ldots, M\} \mapsto\left\{\Phi_{1}, \ldots, \Phi_{\tau}\right\}$ denotes the model of every deployed relay, i.e. $\phi(m) \in\left\{\Phi_{t}\right\}_{t=1}^{\tau} \forall m \in\{1, \ldots, M\}$. The model alphabet $\left\{\Phi_{t}\right\}_{t=1}^{\tau}$ is sorted in ascending order of radius and cost, i.e. $R_{t^{\prime}}>R_{t}$ and $\beta_{t^{\prime}}>\beta_{t}$ if $t^{\prime}>t$. We hereafter define a relay deployment $\mathcal{D}$ as

$$
\mathcal{D} \triangleq\left\{M,\left\{\mathbf{c}_{m}^{R}\right\}_{m=1}^{M},\{\phi(m)\}_{m=1}^{M}\right\},
$$

i.e. as a certain number of deployed relays, their location and model. With these three variables being specified, a $N \times(N+M)$ binary coverage matrix $\mathrm{X}$ can be computed with components $x_{i, j}$ given by

$$
x_{i, j} \triangleq \begin{cases}\mathbb{I}\left(\sqrt{\left(x_{i}-x_{j}\right)^{2}+\left(y_{i}-y_{j}\right)^{2}} \leq \min \left\{r_{i}, r_{j}\right\}\right) & \text { if } 1 \leq j \leq N, \\ \mathbb{I}\left(\sqrt{\left(x_{i}-x_{j-N}^{R}\right)^{2}+\left(y_{i}-y_{j-N}^{R}\right)^{2}} \leq R_{\phi(j-N)}\right) & \text { if } N<j \leq N+M,\end{cases}
$$

where $\mathbb{I}(\cdot)$ is an indicator function taking value 1 if its argument is true. This matrix $\mathbf{X}$ can be computed straightforward from $\left\{\left(x_{i}, y_{i}\right)\right\}_{i=1}^{N},\left\{\left(x_{m}^{R}, y_{m}^{R}\right)\right\}_{m=1}^{M}$ and the coverage radii implicitly expressed by $\{\phi(m)\}_{m=1}^{M}$. This connectivity matrix can be manipulated to yield a more generalized form $\mathbf{X}^{\lambda}$, which denotes a family of $N \times N+M$ multi-hop coverage matrices such that $x_{i, j}^{\lambda}=1$ if there is a connected path of at most $\lambda$ hops from node $i$ to $j$ subject to the connectivity constraint imposed by the minimum between the coverage radii of nodes composing the intermediate links.

As anticipated in the introduction, communications resilience against radio isolation or interrupted radio links is crucial in a disaster situation. A quantitative measure of the resilience of every team deployed in the area can be computed from the above connectivity matrix $\mathbf{X}$ as the normalized fraction of the overall number of nodes through which any given relay is reachable at most $\lambda$ hops. By using the notation introduced in this section, such a measure is given, for node $i \in\{1, \ldots, N\}$ and $\lambda$ hops, by

$$
R^{\lambda}(i) \triangleq \frac{1}{N+M-1} \sum_{\substack{j=1 \\ j \neq i}}^{N+M} \mathbb{I}\left(x_{i, j}=1\right) \mathbb{I}\left(\sum_{m=N+1}^{M} \mathbb{I}\left(x_{j, m}^{\lambda+1}>0\right)\right)
$$

where it has been assumed that $x_{i, i}=1 \forall i$. The value taken by $R^{\lambda}(i)$ will get closer to its maximum as the number of other points that are within $\lambda$ hop coverage range of node $i$ increases. Likewise, the lower the number of $\lambda$-reachable teams from $i$ is, the lower this metric will be. By averaging this value over $N$ a global estimation of the resilience $R_{\text {avg }}^{\lambda}$ can be obtained. 
With this definition in mind, the problem considered aims at discovering the family of Pareto-optimal deployments $\left\{\mathcal{D}_{1}^{*}, \ldots, \mathcal{D}_{K}^{*}\right\}$ such that

$$
\left\{\mathcal{D}_{1}^{*}, \ldots, \mathcal{D}_{K}^{*}\right\}=\underset{\substack{M \\\left\{\mathbf{c}_{m}^{R}\right\}_{m=1}^{M} \\\{\phi(m)\}_{m=1}^{M}}}{\arg \min }\left\{\sum_{n=1}^{N} \mathbb{I}\left(\sum_{m=N+1}^{N+M} x_{n, m}^{\lambda}=0\right), \sum_{m=1}^{M} \beta(\phi(m)), 1-R_{a v g}^{\lambda}\right\}
$$

or equivalently by defining the $\lambda$-coverage $\zeta^{\lambda}(\cdot)$ and total cost $\beta^{T}(\cdot)$ functions,

$$
\begin{aligned}
& \left\{\mathcal{D}_{1}^{*}, \ldots, \mathcal{D}_{K}^{*}\right\} \\
& =\underset{\substack{M \\
\left\{\mathbf{c}_{m}^{R}\right\}_{m=1}^{M} \\
\{\phi(m)\}_{m=1}^{M}}}{\arg \max \zeta^{\lambda}}\left(\left\{\mathbf{c}_{m}^{R}\right\}_{m=1}^{M},\{\phi(m)\}_{m=1}^{M}\right), \min \beta^{T}\left(\{\phi(m)\}_{m=1}^{M}\right), \max R_{a v g}^{\lambda}
\end{aligned}
$$

i.e. a family of $K$ network deployments are sought to Pareto-optimally balance between the achievable coverage $\zeta^{\lambda}\left(\left\{\mathbf{c}_{m}^{R}\right\}_{m=1}^{M},\{\phi(m)\}_{m=1}^{M}\right)$, the total cost of the relays $\beta^{T}\left(\{\phi(m)\}_{m=1}^{M}\right)$ and the radio resilience of the team.

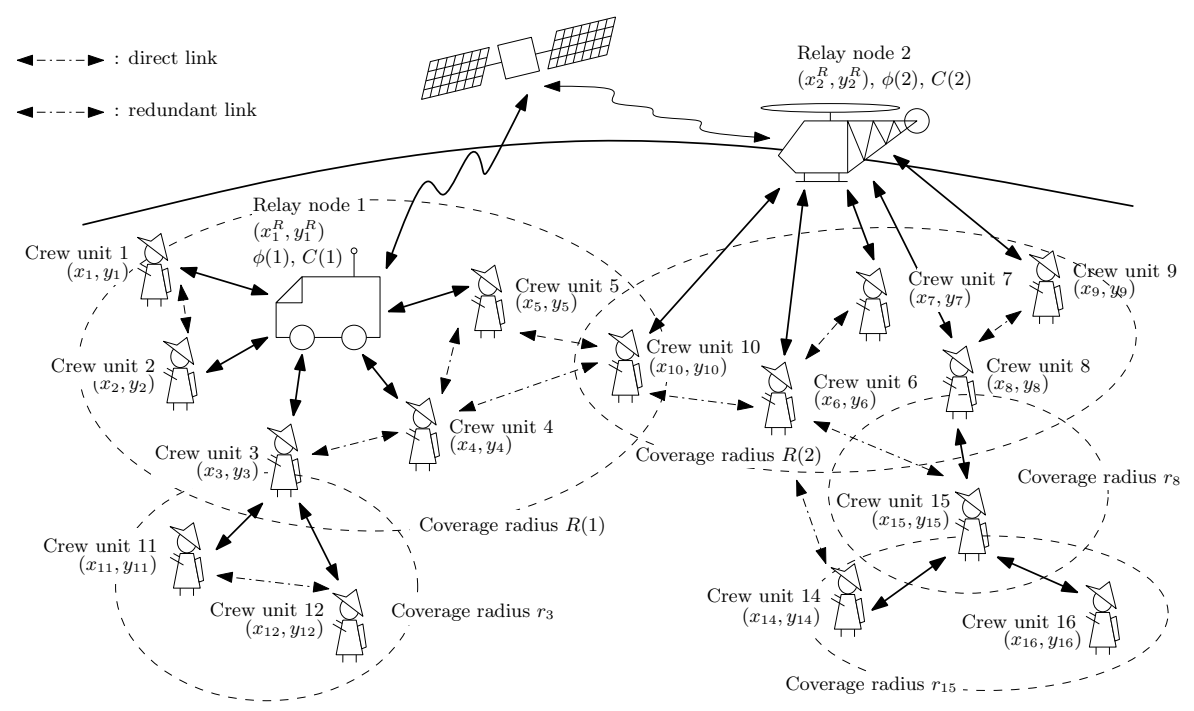

Fig. 1. Multi-hop relaying scenario with redundant links under consideration.

From the operational point of view the produced Pareto-optimal set of deployments will embody an essential information for the decision making when quantifying the maximum achievable coverage given a certain overall budget allocated for the deployment. At this point it is important to note that resilience and coverage can be intuitively thought of as non-conflicting objectives; as the coverage ratio becomes higher, so does the number of achievable redundant teams from a certain location. Based on this rationale, optimally deploying relays under the above three-fold criteria will be reformulated as a bi-objective optimization problem where coverage and resilience will be combined into a single fitness function prioritizing the former over the latter, 
namely,

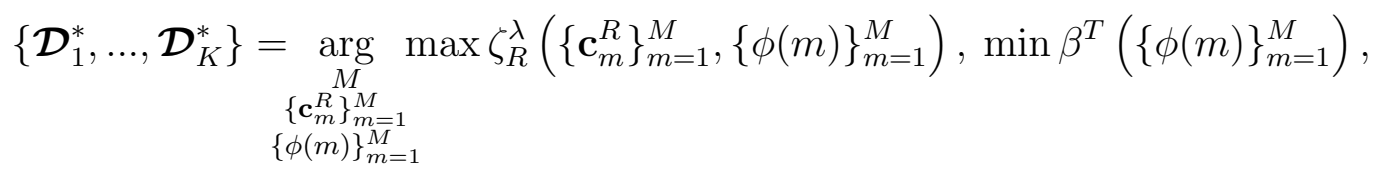

where $\zeta_{R}^{\lambda} \triangleq N^{-1} \sum_{n=1}^{N}\left[\mathbb{I}\left(\sum_{m=N+1}^{N+M} x_{n, m}^{\lambda}>0\right)+R^{\lambda}(n)\right]$, i.e. the averaged sum of the resilience and binary coverage of each node. In words: $\zeta_{R}^{\lambda}$ will reflect a higher priority to covering as many teams as possible with the available budget, and will maximize the communication resilience metric when handling optional deployments with equal coverage and cost values.

\section{Proposed Algorithm}

In order to efficiently tackle the bi-objective relay deployment problem in Expression (4), a novel heuristic scheme based on the Harmony Search (HS) algorithm will be derived and used. First proposed in [71] and subsequently applied to problems arising in diverse knowledge fields [72], HS mimics the progressive harmony enhancement through improvisation and memory attained by jazz musicians in their attempt to arrange an aesthetically good harmony. In a similar fashion to other Evolutionary Computation and Soft Computing optimization techniques [73], HS maintains a population or memory of iteratively refined harmonies (i.e. solutions), on which intelligent permutation and randomization operators resembling the improvisation of musicians are applied to their constituent notes (correspondingly, optimization variables) until a stop criteria is met, e.g. a maximum number of iterations $\mathcal{I}$ is reached.

In regards to the considered scenario, the nominal $\Psi$-sized harmony memory is split into 2 different yet related sub-memories: one for the positions of the relays, and another for their models or types. Since the number of deployed relays may not be the same among the candidates compounding the harmony memory, a maximum number $M_{\max }$ is established as a priori parameter of the algorithm. In order to account for a variable number of deployed relays for each candidate harmony, a new relay model $\Phi_{\varnothing}=(0,0)$ (zero coverage and cost) is included in the family of available relay models $\left\{\Phi_{t}\right\}_{t=1}^{\tau}=\left\{\left(R_{t}, \beta_{t}\right)\right\}_{t=1}^{\tau}$. In this manner, the eventual assignment of $\Phi_{\varnothing}$ to a relay in a given solution will stand for its elimination in the deployment it represents. Therefore, the relay position submemory will be denoted as $\left\{\mathbf{c}^{R}(\psi)\right\}_{\psi=1}^{\Psi}$ with $\mathbf{C}^{R}(\psi) \triangleq\left\{\mathbf{c}_{1}^{R}(\psi), \ldots, \mathbf{c}_{M_{\max }}^{R}(\psi)\right\}$; and $\{\boldsymbol{\phi}(\psi)\}_{\psi=1}^{\Psi}$ standing for the relay model assignment, with $\phi(\psi) \triangleq\left\{\phi(1, \psi), \ldots, \phi\left(M_{\text {max }}, \psi\right)\right\}$. Therefore, for each value $\psi$ of the memory index the represented deployment will be given by $\mathcal{D}(\psi)=\left\{M(\psi), \mathbf{C}^{R}(\psi), \boldsymbol{\phi}(\psi)\right\}$, with $M(\psi)=\sum_{m=1}^{M_{\max }} \mathbb{I}\left(\phi(m, \psi)=\Phi_{\varnothing}\right)$. 


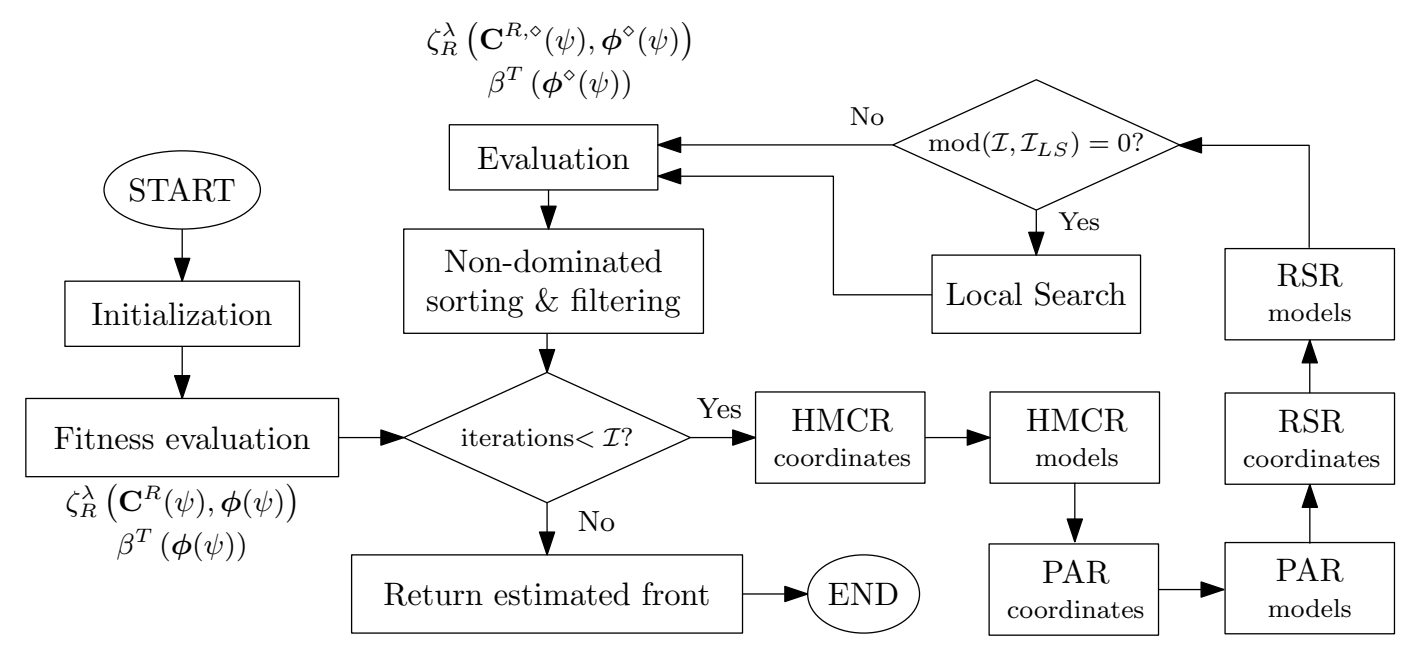

Fig. 2. Flow diagram of the proposed meta-heuristic solver.

In accordance with the diagram in Figure 2, three operators are applied to the harmony memory along iterations:

- Harmony Memory Considering Rate, which denotes the probability that the newly improvised value for a given note is drawn from the values of the same note in the other $\Psi-1$ harmonies in the memory. In the proposed scheme this operator discloses two independently applied HMCR processes (one per sub-memory), driven by probabilistic parameters $\mathrm{HMCR}^{C}$ (coordinates) and $\mathrm{HMCR}^{M}$ (models), both defined in $\mathbb{R}[0,1]$. The former is always applied disregarding the model to which the relays are assigned, whereas the later refines not only the model of the relays, but also its effective number $M(\psi)$.

At this point it is important to note that since no ordering of the harmony memory is done, the HMCR operator may yield a too explorative search due to the fact that relays located at a given position $m$ in candidate harmonies $\psi^{\prime}$ and $\psi^{\prime \prime}$ within the harmony memory could not correspond in practice to the same relay. In this same line of reasoning, two positions $m$ and $m^{\prime}$ might represent the same relay, yet would not undergo together the same HMCR procedure. To overcome this lack of alignment, the naive definition of the HMCR operator is modified to operate as follows: when it is to be applied to a component of either the coordinates or models - e.g. $\mathbf{c}_{m}^{R}(\psi)-\mathrm{a}$ distance-dependent dictionary of possible replacing relays is composed, one per each of the rest of candidate harmonies. As such, the candidate harmony $\psi^{\prime} \neq \psi$ will contribute to this dictionary by checking, first, whether any of its compounding relays is closer to the one to be replaced by less than $\min \left\{R_{1}, \ldots, R_{\tau}\right\}$. If so, the relay in question will be set as the candidate relay for replacement given by this harmony. Otherwise the operator proceeds as usual and chooses $\mathbf{c}_{m}^{R}\left(\psi^{\prime}\right)$ as a potential replacement of the relay. This distance-dependent selection criterion is expected to accelerate the convergence of the algorithm due to a better aligned HMCR operator.

- Pitch Adjusting Rate, which establishes the probability that the value of a 
given note is replaced with any of its neighboring values in its corresponding alphabet. Due to the alphabets of the above two sub-memories being continuous (coordinates) and discrete (models), it is necessary to particularize this operator for every sub-memory. When dealing with the coordinates sub-memory, this process, driven by probability $\mathrm{PAR}^{C}$, is given by

$$
\mathbf{c}_{m}^{R, \diamond}(\psi) \triangleq\left(x_{m}^{R, \diamond}(\psi), y_{m}^{R, \diamond}(\psi)\right)=\left\{\begin{array}{l}
\left(x_{m}^{R}(\psi), y_{m}^{R}(\psi)\right)+\mathbf{z}_{\varepsilon} \text { w.prob. } \text { PAR }^{C} \\
\left(x_{m}^{R}(\psi), y_{m}^{R}(\psi)\right) \quad \text { w.prob. 1-PAR }
\end{array}\right.
$$

where the superscript ${ }^{\diamond}$ denotes the new coordinate value, $\epsilon \in \mathbb{R}^{+}$is referred to as the pitch adjustment bandwidth, and $\mathbf{z}_{\beta}$ is the realization of a twodimensional uniform random variable with continuous support in the range $[-\varepsilon, \varepsilon] \times[-\varepsilon, \varepsilon]$. As for the model sub-memory, the probabilistic parameter $\operatorname{PAR}^{M}$ will set the probability that the new value $\phi^{\diamond}(m, \psi)$ for note $\phi(m, \psi)$ is taken at random from its higher and lower neighboring values in the alphabet $\{\varnothing, 1, \ldots, \tau\}$, where $\tau$ represents the number of relay models.

- Random Selection Rate: the probabilistic parameters $\operatorname{RSR}^{C}$ (coordinates) and $\mathrm{RSR}^{M}$ (models) set the probability that the new value for a given note will be drawn uniformly at random (i.e. without any neighborhood consideration) from its corresponding alphabet.

These operators are sequentially applied to each note of every harmony kept in both sub-memories. Once applied over the entire set of notes, the fitness functions $\zeta_{R}^{\lambda}\left(\mathbf{C}^{R, \diamond}(\psi), \phi^{\diamond}(\psi)\right)$ and $\beta^{T}\left(\phi^{\diamond}(\psi)\right)$ of the newly improvised harmonies $\left.\mathcal{D}^{\diamond}(\psi)\right\}_{\psi=1}^{\Psi}$ are evaluated, based on which both the new harmonies and those remaining from the previous iteration are ordered and filtered following a dual selection hinging on Pareto-dominance ranking and crowding distance. To be specific, each harmony is scored with a numerical rank equal to its non-dominance level (namely, 1 for the best non-dominated level, 2 for the next best level, etc). Once all fronts have been ranked, a measure representing the sum of distances to the closest harmony along each metric establishes an ordering among the solutions belonging to a certain rank: harmonies with large crowding distance are preferential with respect to solutions with small distance. Finally only the best $\Psi$ harmonies (considering first the ordering among the fronts and then the one among the harmonies) are kept in the harmony memory for the next iteration.

In order to improve the convergence of the search algorithm, a computationally lightweight local search procedure is inserted into the main algorithmic thread of the proposed solver. The local search method aims at enhancing the algorithm by providing topological information to the global search process in its early running stages. To this end, every $\mathcal{I}_{L S} \ll \mathcal{I}$ iterations a crisp clustering scheme is applied to the space spanned by the coordinates of the deployed teams $\left\{\mathbf{c}_{n}\right\}_{n=1}^{N}$, using, as an input, its effective number of relays $M(\psi)$. This 
produces a set of $M(\psi)$ centroids $\left\{\mathbf{c}_{m}^{\nabla}\right\}_{m=1}^{M(\psi)}$ for candidate harmony $\psi$. These centroids could guide the solution of the algorithm towards less sparsely populated regions of the disaster area, however they are not aware of the multi-hop nature of the communications to be deployed, nor are they sensible to the limited coverage radii of the relay models and the resilience fitness included in $\zeta_{R}^{\lambda}(\cdot)$. Consequently, each of the relay coordinates in harmony $\psi$ is replaced by the those of the mid point in the line that connects it to the closest relay produced by the clustering algorithm. This ensures that the algorithm does not get biased by the lack of awareness of the local search procedure with respect to the particularities of the problem.

\section{Experimental Results and Discussion}

Several experiments have been carried out towards assessing the performance of the proposed scheme when applied over synthetically generated scenarios, as well as over setups of increased realism and complexity. The first pool of simulations can be conceived as a benchmark aimed at comparatively assessing the performance of different schemes in scenarios of varying complexity:

(1) A naive scheme composed by a classical distance-based clustering technique with estimation of the number of clusters (X-Means [74]), followed by an exhaustive evaluation of all model combinations. This approach will be denoted as CEX.

(2) A genetic algorithm with Roulette Wheel selection, uniform crossover (rates $P_{m}^{C}$ and $P_{m}^{M}$ ) and standard mutation (correspondingly, $P_{c}^{C}$ and $P_{c}^{M}$ ) operators with no local search. The focus is placed on verifying whether the HS operators provide any performance gain in terms of Pareto optimality with respect to those of the GA approach. We will label this scheme as GA.

(3) The HS based solver presented in this paper, with the standard HMCR operator and no local search included (referred to as HS).

(4) The HS based solver with the HMCR operator modified to account for the proximity of misaligned relays within the harmony memory (HS+HMCR).

(5) The HS based approach with the modified HMCR operator and incorporating the clustering-based local search procedure. Specifically the socalled Mini Batch K-Means [75] will be utilized (HS+HMCR+LS).

Specifically, $N \in\{100,300,500\}$ brigade units are uniformly spread at random over a square area $A$ of dimensions $1000 \times 1000$, each having a personal radio equipment with coverage $r_{n}=r=100$. Each of the proposed algorithms selects the number of relays $M \leq M_{\max }=30$, their positions in $A$ and models from $\left\{\Phi_{t}\right\}_{t=1}^{6}$, with $\left\{R_{t}\right\}_{t=1}^{6}=\{50,100,150,200,250,300\}$ denoting their radii and $\left\{\beta_{t}\right\}_{t=1}^{6}=\{100,300,700,1100,1800,3000\}$ m.u. (monetary units) 
their costs. The main purpose of the performed simulations is 1) to check out that the set of produced deployments effectively balance between cost and coverage; and 2) to verify that as the maximum number of hops $\lambda$ is made higher, multi-hop relaying enhances the coverage statistics of the produced deployment for a given overall cost range. To this end, different cases with $\lambda \in\{2,3,4\}$ are discussed in this section. It is important to notice that $\lambda=1$ stands for the scenario where only direct connections to the deployed relays are allowed. Regarding the parameters controlling the underlying HS-based search algorithm, a memory size of $\Psi=20$ harmonies is utilized in all cases, with values $H M C R^{C}=H M C R^{M}=0.5, P A R^{C}=P A R^{M}=0.1, R S R^{C}=$ $R S R^{M}=0.05$ and $\epsilon=50$ optimized by exhaustive search over a value grid ${ }^{1}$ (not included for the sake of space). A similar parameter tuning procedure has been followed for the GA approach, fixing in all cases the population size equal to 20 (for the sake of fairness in terms of fitness evaluations) and yielding $P_{m}^{C}=0.6, P_{m}^{M}=0.4, P_{c}^{C}=0.1$ and $P_{c}^{M}=0.05$. A total of $\mathcal{I}=200$ iterations have been set for each of the 20 experiments run for every $\lambda$. Comparisons will be done in terms of Pareto optimality measured by the so-called hypervolume index $I_{h v}$ [76]. The reference for the computation of this indicator will be given by the absolute best Pareto front when aggregating the results for all the compared algorithms. Statistics (mean, standard deviation) of $I_{h v}$ over the Monte Carlo experiments will be analyzed.

The discussion begins by analyzing Table 1, which shows the mean and standard deviation statistics of the hypervolume ratio (in \% over the reference point) achieved by each of the compared schemes: the higher this ratio is, the wider the area covered by the Pareto front will be. First it is interesting to observe that as the number of deployed teams in the ground increases, gaps between the compared techniques become smaller as $\lambda$ (i.e. the maximum number of admissible hops) increases. The reason being that as the area under study becomes more populated, multi-hop networking allows a more effective coverage even if the optimal locations for the relays are not accurately estimated. Nevertheless, the obtained results evince that the proposed HS-based algorithm incorporating the special distance-based HMCR operator and the local search method outperform any other scheme in terms of Pareto optimality. Figure 3.a depicts graphically the Pareto dominance of the pro-

1 In essence each point in this grid corresponds to a certain combination of values for the parameters driving the algorithm at hand. For instance, in the $\mid$ HS | approach this search would be performed over a 6-dimensional grid with points $\left\{H M C R^{C}, H M C R^{M}, P A R^{C}, P A R^{M}, R S R^{C}, R S R^{M}\right\}$ forming a irregular mesh with different spacing per dimension, the latter being given by the sampling interval of the values for each parameter. The average performance of the algorithm with its parameters set as dictated at every point and measured over three synthetic scenarios with $\lambda=\{2,3,4\}$ is what determines the optimal parameter setting: the point scoring best among all points in the mesh. 
posed algorithm by plotting together the aggregated Pareto front produced by each scheme for $\lambda=3$ hops and $N=300$ points to be covered. The analysis follows in Figure 3.b, where the overall estimated Pareto-optimal deployments produced by the algorithm in cases $\lambda=2$ to $\lambda=4$ are depicted. As seen in the plot the higher the admissible number of hops $\lambda$ is, the higher the achievable coverage for a given cost results to be, which can be intuitively expected due to the extended coverage granted by intermediate brigades lying less than $\lambda$ hops away from the relay node.

For graphically illustrating the set of produced relay deployments, in Figure 3.b two points (marked with $\bigcirc$ ) have been chosen: 1 ) $\lambda=3$ hops, $\beta^{T}=500$ m.u. and $45.43 \%$ coverage; and 2) $\lambda=4$ hops, $\beta^{T}=7800$ m.u. and $100 \%$ coverage. The relay deployments corresponding to these points are depicted in Figures 3.c and 3.d. Coverage radii corresponding to covered brigade units $(\bullet)$ and deployed relays $(\square)$ are depicted with light and bold dashed circles, respectively. As can be seen in these plots, the proposed scheme is able to deploy relays under different - yet Pareto optimal - balances between the coverage and cost of the deployment.

Table 1

Performance statistics (mean \pm standard deviation) for the hypervolume $I_{h v}$ (in $\%$ with respect to the reference point) for synthetic scenarios with varying $N$.

\begin{tabular}{|c|c|c|c|c|c|c|}
\hline$N$ & Hops & CEX & GA & HS & HS+HMCR & HS+HMCR+LS \\
\hline \multirow{2}{*}{$\&$} & $\lambda=2$ & $53.1 \pm 4.4$ & $78.3 \pm 3.9$ & $80.4 \pm 1.7$ & $82.5 \pm 2.2$ & $84.9 \pm 3.2$ \\
\cline { 2 - 7 } & $\lambda=3$ & $58.5 \pm 5.6$ & $82.2 \pm 2.8$ & $84.1 \pm 2.1$ & $86.6 \pm 1.9$ & $88.7 \pm 3.6$ \\
\cline { 2 - 7 } & $\lambda=4$ & $62.7 \pm 5.1$ & $86.4 \pm 2.2$ & $89.0 \pm 2.4$ & $91.3 \pm 1.8$ & $94.1 \pm 2.8$ \\
\hline \multirow{2}{*}{ \& } & $\lambda=2$ & $60.2 \pm 4.3$ & $80.1 \pm 3.5$ & $82.7 \pm 2.2$ & $85.1 \pm 2.1$ & $86.6 \pm 2.7$ \\
\cline { 2 - 7 } & $\lambda=3$ & $64.5 \pm 4.1$ & $82.2 \pm 2.7$ & $83.8 \pm 2.0$ & $87.7 \pm 1.9$ & $90.8 \pm 2.2$ \\
\hline \multirow{2}{*}{$\&$} & $\lambda=4$ & $70.1 \pm 3.7$ & $85.9 \pm 2.6$ & $89.2 \pm 1.8$ & $92.9 \pm 2.1$ & $95.6 \pm 1.1$ \\
\cline { 2 - 7 } & $\lambda=2$ & $67.4 \pm 4.2$ & $85.4 \pm 1.9$ & $87.1 \pm 2.4$ & $89.5 \pm 1.4$ & $90.8 \pm 1.6$ \\
\cline { 2 - 7 } & $\lambda=4$ & $71.9 \pm 3.3$ & $90.3 \pm 2.1$ & $92.3 \pm 2.0$ & $94.6 \pm 1.3$ & $95.1 \pm 1.5$ \\
\hline
\end{tabular}

Once the HS+HMCR+LS approach has been shown to outperform the rest of algorithms, a second simulation will evince the applicability of this proposed solver to more realistic scenarios with non-necessarily circular coverage areas. To this end, we have processed open LIDAR data made available by the Open Data Euskadi initiative [77]. The capturing campaign was held between July and August 2012, and covered the entire region of the Basque Country with a resolution of 0.5 points per square meter. A fraction of the entire dataset was 
trimmed to focus on an hypothesized relay deployment in the center of the city of Bilbao, Spain (see Figures 4.a and 4.b). The considered grid spans a square area of 4 square kilometers, with irregular orography and high density of buildings that make the circular coverage model utilized far from realistic.

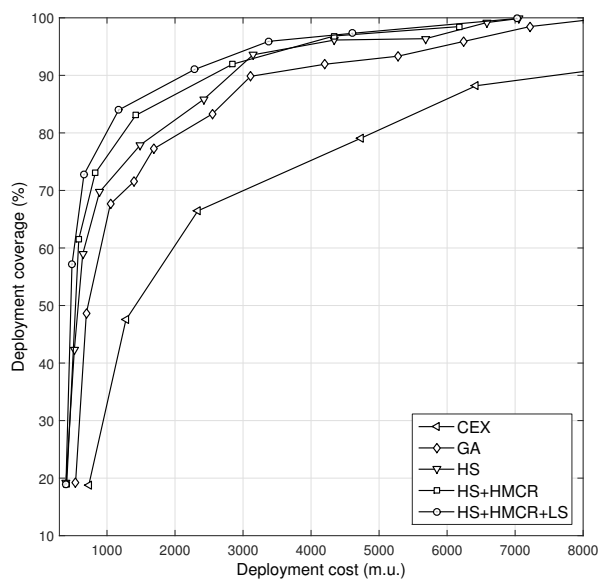

(a)

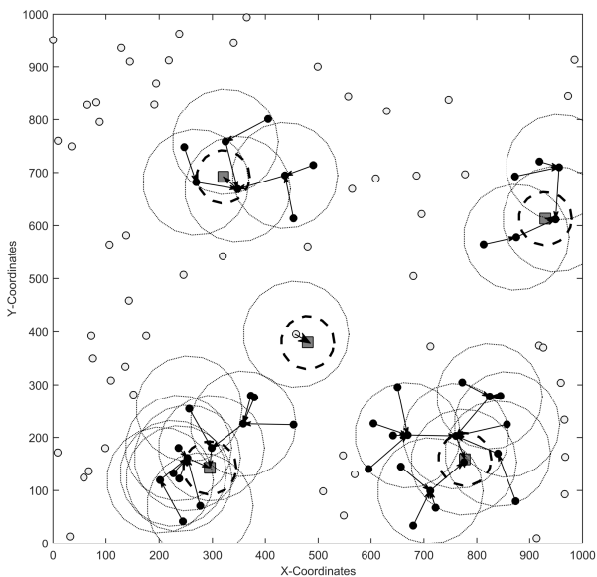

(c)

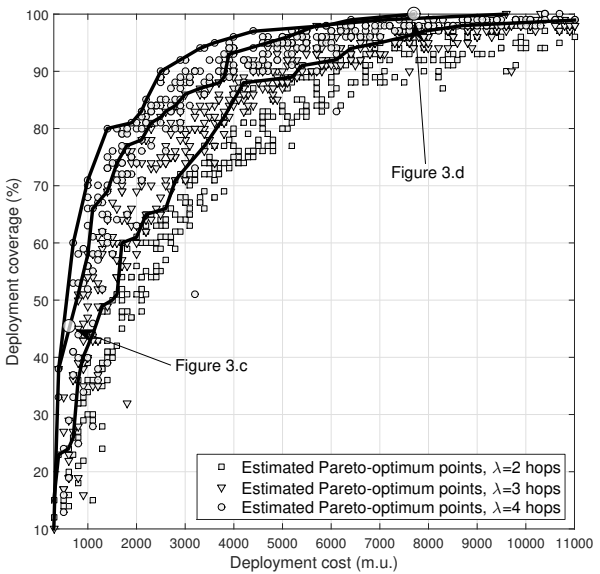

(b)

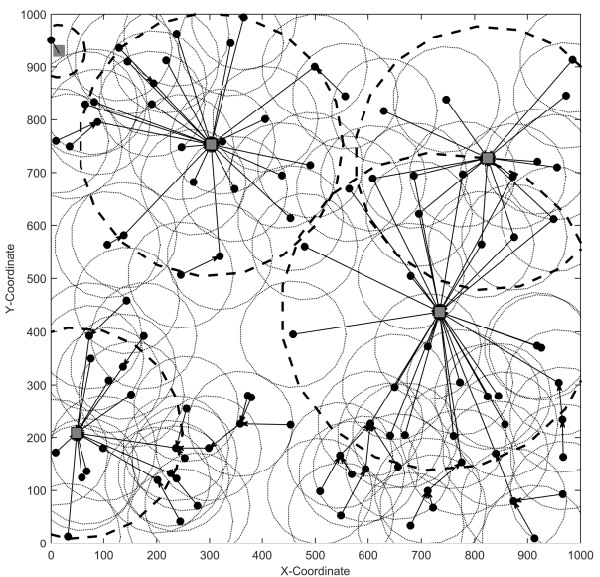

(d)

Fig. 3. (a) Aggregated Pareto fronts inferred by each scheme under comparison for $\lambda=3$ and $N=300$; (b) Comparison of different Pareto fronts for the HS+HMCR+LS approach, $N=100$ and different values of $\lambda$; (c) Relay deployment corresponding to a $45.43 \%$ coverage rate and 500 m.u. for a maximum of $\lambda=3$ allowed hops; (d) Relay deployment corresponding to a $100 \%$ coverage rate and 7800 m.u. for $\lambda=4$.

Figures 5.a and 5.b depict the deployments corresponding to the extreme points of the Pareto point obtained for $\lambda=3$ hops and 100 points uniformly spread over the parts classified as "terrain" in the dataset. Maximum (i.e. free line of sight assuming antennas at 2.5 meters above ground) coverage radii have been set to $r_{n}=r=150$ meters (brigades) and $\left\{R_{t}\right\}_{t=1}^{6}=$ $\{150,200,400,600,800,1000\}$ meters (relays). Red lines delimit the line-ofsight coverage area of the deployed relays. As shown in these plots and evinced by their corresponding metric values, the proposed HS+HMCR+LS approach is 
able to trade one objective for the other by properly setting the relay number, locations and models.

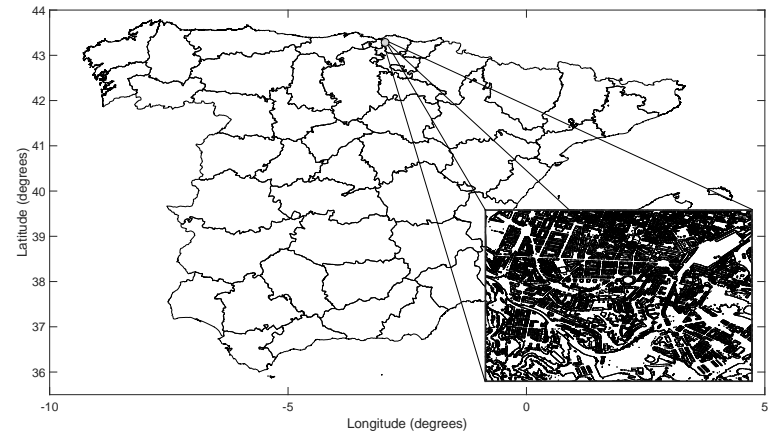

(a)

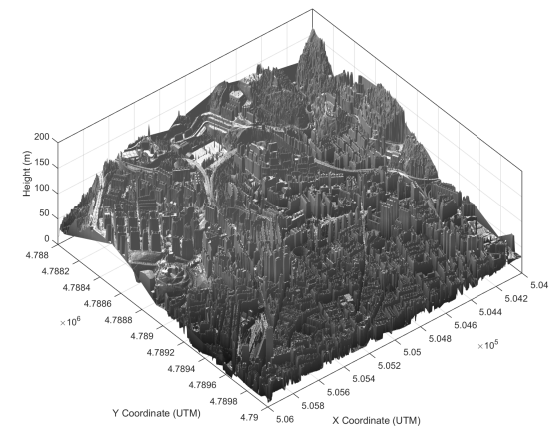

(b)

Fig. 4. (a) Location of the city of Bilbao where the realistic set of experiments is performed; (b) an overview of the LIDAR grid after trimming the area of interest.

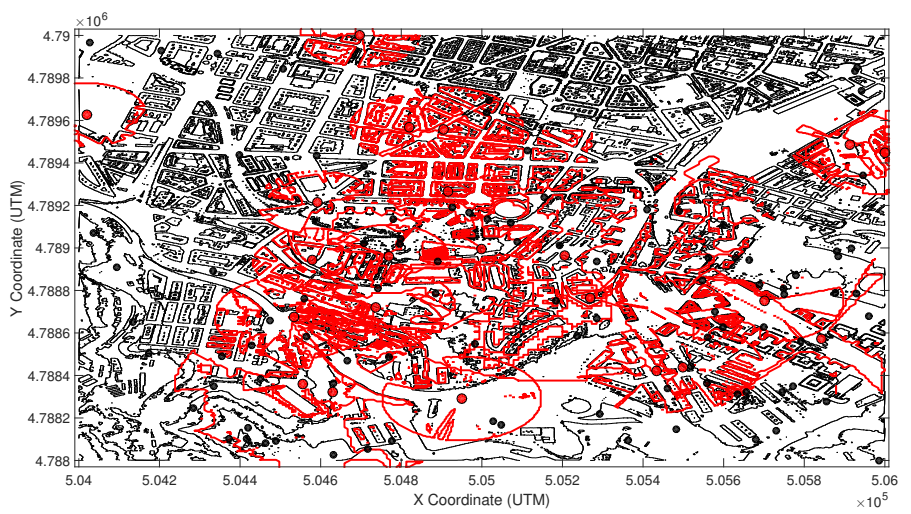

(a)

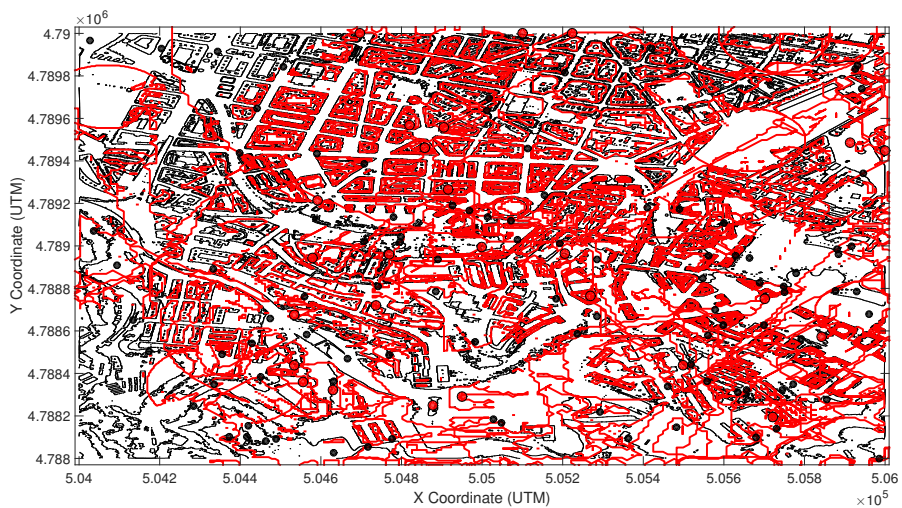

(b)

Fig. 5. Contour plot showing (a) the relay deployment for $\lambda=3$ hops corresponding to the extreme Pareto point $\left(\zeta_{R}^{\lambda}, \beta^{T}\right)=(54.02 \%, 6000$ m.u. $)$ ( $M=22$ relays); (b) relay deployment corresponding to the other extreme point $\left(\zeta_{R}^{\lambda}, \beta^{T}\right)=(90.05 \%, 31300$ m.u. $)(M=26$ relays $)$. Black points correspond to the brigades on ground, whereas red markers denote the deployed relays. 


\section{Conclusions and Future Work}

This paper has elaborated on the deployment of wireless communication relays over disaster areas, a resource allocation task of inherent difficulty when it is undertaken in a economically constrained context. When nodes are allowed to communicate with the relays via multi-hop wireless networking, the problem gets even harder due to the need for simultaneously considering cost, coverage and resilience against link failures. This work has formulated a bi-objective optimization problem, jointly addressing this three-fold criteria. To efficiently deal with this problem, a bi-objective meta-heuristic algorithm based on the Harmony Search paradigm has been proposed, to yield the Pareto-optimal set of heterogeneous relay deployments that differently balance a joint coverageresilience metric and the economical cost of the deployment. The proposed Harmony Search approach iteratively refine the position and models of the deployed relays to obtain an optimal solution. The number of required relays is obtained by an additional relay model that allows for deleting and adding relays during the search procedure. Simulations performed on scenarios with increasing values of the admissible number of communication hops $\lambda$ have shed light on the benefits and capabilities of the proposed tool, which provides the operations commander with crucial information on the maximum achievable coverage given the available budget for the deployment. The enhanced Pareto optimality attained by the proposed HS-based approach in comparison to the other schemes in this benchmark is deemed crucial in the current globally constrained economical situation, where strong financial cuts require authorities to allocate their resources as cost-effectively as possible.

Future research will consider the mobility of the teams on the ground and their predicted routes / operations area in the coverage metric. Energy efficiency and autonomy of the relays will be also analyzed, specially in what regards to the user equipment of teams that perform relaying at intermediate hops for high values of $\lambda$.

\section{References}

[1] S. Solomon, D. Qin, M. Manning, M. Marquis, K. Averyt, M. M. B. Tignor, H. L. Miller Jr., Z. Chen, "Climate Change 2007: The Physical Science Basis", Cambridge University Press, 996 pp, 2007.

[2] L. M. Bouwer, "Have Disaster Losses Increased Due to Anthropogenic Climate Change?", Bulletin of the American Meteorological Society, Vol. 92, pp. 39-46, 2011.

[3] S. Salcedo-Sanz, R. C. Deo, L. Carro-Calvo, B. Saavedra-Moreno, "Monthly Prediction of Air Temperature in Australia and New Zealand with Machine Learning Algorithms", Theoretical and Applied Climatology, in press, 2015. 
[4] M. I. Chidean, J. Muñoz-Bulnes, J. Ramiro-Bargueño, A. Caamaño-Fernández and S. Salcedo-Sanz, "Spatio-temporal trend analysis of air temperature in Europe and western Asia using data-coupled clustering" Global and Planetary Change, vol. 129, pp. 45-55, 2015.

[5] Y. Liu, J. Stanturf, S. Goodrick, "Trends in Global Wildfire Potential in a Changing Climate", Forest Ecology and Management, Vol. 259, pp. 685-697, 2010 .

[6] T. Ghose, "Climate Change May Be Worsening Western Wildfires", Livescience, http://www.livescience.com/41877-western-wildfires-getting-worse. html. Retrieved in December 2013

[7] T. McGhee, "4,167 Colorado Wildfires caused Record Losses of $\$ 538$ Million in 2012", The Denver Post, 2013.

[8] "Hyogo Framework for Action 2005-2015: Building the Resilience of Nations and Communities to Disasters", United Nations Office for Disaster Risk Reduction, 2007.

[9] "Sendai Framework for Disaster Risk Reduction 2015-2030", UN World Conference on Disaster Risk Reduction, Sendai, Japan, 2015.

[10] A. Aitsi-Selmi, V. Murray, "The Sendai Framework: Disaster Risk Reduction through a Health Lens'", Bulletin of the World Health Organization, Vol. 93, N. 6, 2015.

[11] "ASEAN Agreement on Disaster Management and Emergency Responce Work Programme for 2010-2015", Association of Southeast Asian Nations, 2009.

[12] P. Yi, Y. Zhang, Y. Tang, S. Li, "An Incident Information Management Framework based on Data Integration, Data Mining, and Multi-Criteria Decision Making", Decision Support Systems, Vol. 51, pp. 316-327, 2011.

[13] L. Yu, K. Lai, "A Distance-based Group Decision-making Methodology for Multi-person Multi-criteria Emergency Decision Support", Decision Support Systems, Vol. 51, pp. 307-315, 2011.

[14] Arizona State Forestry Division: Yarnell Hill Fire Serious Accident Investigation Report, 2013.

[15] V. Pons i Grau, "La Explosión del Monte: El Trágico Suceso acaecido durante el Incendio Forestal de Guadalajara" (in Spanish). Imprenta Romeu, 2008.

[16] W. Jendsch, "Das große Feuer, Jendsch Feuerwehrpresse" (Fire Press, in German), Fachbeitrag (technical report) 2727/98, 1998.

[17] R. T. Newkirk, "The Increasing Cost of Disasters in Developed Countries: A Challenge to Local Planning and Government", Journal of Contingencies and Crisis Management, Vol. 9, N. 3, pp. 159-170, 2001.

[18] "Foro Ambiental de Castilla-La Mancha: Valoración de la Campaña 2013 de Incendios forestales en Castilla-La Mancha" (in Spanish), 2013. 
[19] M. A. Ertem, N. Buyurgan, "An Auction-based Framework for Resource Allocation in Disaster Relief", Journal of Humanitarian Logistics and Supply Chain Management, Vol. 1, N. 2, pp. 170-188, 2011.

[20] N. Liu, Y. Ye, "Humanitarian Logistics Planning for Natural Disaster Response with Bayesian Information Updates", Journal of Industrial and Management Optimization, Vol. 10, N. 3, pp. 665-689, 2014.

[21] F. Wex, G. Schryen, S. Feuerriegel, D. Neumann, "Emergency Response in Natural Disaster Management: Allocation and Scheduling of Rescue Units", European Journal of Operational Research, Vol. 235, N. 3, pp. 697-708, 2014.

[22] R. Minciardi, R. Sacile, E. Trasforini, "Resource Allocation in Integrated Preoperational and Operational Management of Natural Hazards", Risk Analysis, Vol. 29, N. 1, pp. 62-75, 2009.

[23] A. Dodo, R. A. Davidson, N. X. Xu, L. K. Nozick, "Application of Regional Earthquake Mitigation Optimization", Computers \& Operations Research, Vol. 34, N. 8, pp. 2478-2494, 2007.

[24] M.-S. Chang, Y. L. Tseng, J.-W. Chen, "A Scenario Planning Approach for the Flood Emergency Logistics Preparation Problem under Uncertainty", Transportation Research Part E: Logistics and Transportation Review, Vol. 43, N. 6, pp. 737-754, 2007.

[25] Y.-J. Zheng, H.-F. Ling, "Emergency Transportation Planning in Disaster Relief Supply Chain Management: a Cooperative Fuzzy Optimization Approach", Soft Computing, Vol. 17, N. 7, pp. 1301-1314, 2013.

[26] K. Onan, F. Ülengin, B. Sennaroglu, "An Evolutionary Multi-objective Optimization Approach to Disaster Waste Management: A Case Study of Istanbul, Turkey", Expert Systems with Applications, Vol. 42, N. 22, pp. 8850$8857,2015$.

[27] C. T. Zahn, "Black Box Maximization of Circular Coverage", Journal of Research of the National Bureau Standards B, Vol. 66, pp. 181-216, 1962.

[28] D. S. Johnson, "The NP-Completeness Column: An Ongoing Guide", Journal of Algorithms, Vol. 3, N. 2, pp. 182-195, 1982.

[29] D. S. Houchbaum, W. Maass, "Approximation Schemes for Covering and Packing Problems in Image Processing and VLSI", Journal of the ACM, Vol. 32, N. 1, pp. 130-136, 1985.

[30] W. Guo, X. Huang, L. Liu, "Dynamic Relay Deployment for Disaster Area Wireless Networks", Wireless Communications and Mobile Computing, Vol. 10, N. 9, pp. 1238-1252, 2010.

[31] A. Agnetis, E. Grande, P. B. Mirchandani, A. Pacifici, "Covering a Line Segment with Variable Radius Discs", Computers \& Operations Research, Vol. 36, N. 5, pp. 1423-1436, 2009. 
[32] M. N. Bilbao, J. Del Ser, S. Salcedo-Sanz, C. Casanova-Mateo, "On the Application of Multi-Objective Harmony Search Heuristics to the Predictive Deployment of Firefighting Aircrafts: a Realistic Case Study", International Journal of Bio-Inspired Computation, Vol. 7, N. 5, pp. 270-284, 2015.

[33] M. N. Bilbao, S. Gil-Lopez, J. Del Ser, S. Salcedo-Sanz, M. Sanchez-Ponte, A. Arana-Castro, "Novel Hybrid Heuristics for an Extension of the Dynamic Relay Deployment Problem over Disaster Areas", TOP, Vol. 22, N. 3, pp. 997-1016, 2014.

[34] M. N. Bilbao, J. Del Ser, S. Salcedo-Sanz, S, Gil-Lopez, J. A. PortillaFigueras, "A Bi-objective Harmony Search Approach for Deploying CostEffective Multi-hop Communications over Large-Area Wildfires", International Joint Conference SOCO'14-CISIS'14-ICEUTE'14, pp. 93-103, 2014.

[35] D. M. West, E. Valentini, "How Mobile Devices are Transforming Disaster Relief and Public Safety", Center for Technology Innovation at Brookings (Issues in Technology Innovation), White Paper, 2013.

[36] R. Ferrus, O. Sallent, G. Baldini, L. Goratti, "LTE: the technology driver for future public safety communications", IEEE Communications Magazine, Vol.51, N. 10, pp. 154-161, 2013.

[37] D. G. Reina, M. Askalani, et al., "A Survey on Multihop Ad Hoc Networks for Disaster Response Scenarios", International Journal of Distributed Sensor Networks, Vol. 2015, 16 pages, 2015.

[38] E. Amaldi, A. Capone, M. Cesana, I. Filippini, F. Malucelli, "Optimization Models and Methods for Planning Wireless Mesh Networks", Computer Networks, Vol. 52, N. 11, pp. 2159-2171, 2008.

[39] M. Younis, K. Akkaya, "Strategies and Techniques for Node Placement in Wireless Sensor Networks: A Survey", Ad Hoc Networks, Vol. 6, N. 4, pp. 621-655, 2008.

[40] D. Benyamina, A. Hafid, M. Gendreau, "Wireless Mesh Networks Design - A Survey", IEEE Communications Surveys Tutorials, Vol. 14, N. 2, pp. 299-310, 2012 .

[41] P. H. Pathak, R. Dutta, "A Survey of Network Design Problems and Joint Design Approaches in Wireless Mesh Networks", IEEE Communications Surveys Tutorials, Vol. 13, N. 3, pp. 396-428, 2011.

[42] S. G. Merlin and A. Nachiappan, "A Review on Optimal Node Placement Methods in Wireless Mesh Network Planning" i-Manager's Journal on Wireless Communication Networks, Vol. 3, N. 1, pp. 35-40, 2014.

[43] I. Khou, P. Minet, A. Laouiti, S. Mahfoudh, "Survey of Deployment Algorithms in Wireless Sensor Networks: Coverage and Connectivity Issues and Challenges", International Journal of Autonomous and Adaptive Communications Systems, p. 24, 2014. 
[44] M. A. Adnan, M. A. Razzaque, I. Ahmed, I. F. Isnin, "Bio-Mimic Optimization Strategies in Wireless Sensor Networks: A Survey", Sensors, Vol. 14, N. 1, pp. 299-345, 2014.

[45] D. Benyamina, A. Hafid, M. Gendreau, "Optimal Placement of Gateways in Multi-hop Wireless Mesh Networks: A Clustering-Based Approach", IEEE Conference on Local Computer Networks (LCN), pp. 625-632, 2009.

[46] D. N. Le, "A Comparative Study of Gateway Placement Optimization in Wireless Mesh Network using GA, PSO and ACO", International Journal of Information \& Network Security, Vol. 2, N. 4, pp. 292-304, 2013.

[47] G. De Marco, "MOGAMESH: A Multi-Objective Algorithm for Node Placement in Wireless Mesh Networks based on Genetic Algorithms", International Symposium on Wireless Communication Systems, pp. 388-392, 2009 .

[48] J. M. Lanza, J.A. Gomez, "Assuming Multiobjective Metaheuristics to Solve a Three-Objective Optimization Problem for Relay Node Deployment in Wireless Sensor Networks", Applied Soft Computing Vol. 30(C), pp. 675-687, 2015.

[49] Y. Cai, Y. Xia, L. Dai, C. Xiao, J. Wu, "Coordination of Repeaters based on Simulated Annealing Algorithm and Monte-Carlo Algorithm", Neurocomputing, Vol. 97, pp. 9-15, 2012.

[50] M. Ikeda, T. Oda, S. Sakamoto, T. Honda, L. Barolli, "Analysis of WMN-SA and WMN-GA Simulation Results: A Comparison Performance for Wireless Mesh Networks", International Conference on Network-Based Information Systems (NBiS), pp. 45-52, 2014.

[51] D. Elmazi, T. Oda, S. Sakamoto, T. Honda, E. Spaho, L. Barolli, F. Xhafa, "Friedman Test for Analysing WMNs: A Comparison Study for Genetic Algorithms and Simulated Annealing", International Conference on Innovative Mobile and Internet Services in Ubiquitous Computing, pp. 171-178, 2015.

[52] M. Di Felice, L. Bedogni, et al, "Smartphones Like Stem Cells: Cooperation and Evolution for Emergency Communication in Post-disaster Scenarios", International Black Sea Conference on Communications and Networking (BlackSeaCom), pp. 28-33, 2013.

[53] T. Pfeiffenberger, P. Dorfinger, F. von Tullenburg, "Communication Coverage Awareness for Self-aligning Wireless Communication in Disaster Operations", IEEE International Conference on Pervasive Computing and Communication Workshops, pp. 481-486, 2015.

[54] X. S. Yang, "Firefly algorithm, stochastic test functions and design optimisation", International Journal of Bio-Inspired Computation, Vol. 2(2), pp. 78-84, 2010.

[55] M. Xu, G. Liu, "A Multipopulation Firefly Algorithm for Correlated Data Routing in Underwater Wireless Sensor Networks", International Journal of Distributed Sensor Networks, 2013. 
[56] E. S. Kumar, "Fire-LEACH: A Novel Clustering Protocol for Wireless Sensor Networks based on Firefly Algorithm", International Journal of Computer Science Theory and Application, Vol. 1(1), pp. 12-17, 2014.

[57] S. Cao, J. Wang, X. Gu, "A Wireless Sensor Network Location Algorithm based on Firefly Algorithm", AsiaSim 2012, pp. 18-26, 2012.

[58] X. S. Yang, S. Deb, "Cuckoo Search via Lévy Flights", World Congress on Nature \& Biologically Inspired Computing, pp. 210-214, 2009.

[59] M. Dhivya, M. Sundarambal, "Cuckoo Search for Data Gathering in Wireless Sensor Networks", International Journal of Mobile Communications, Vol. 9(6), pp. $642-656,2011$.

[60] S. Goyal, M. S. Patterh, "Wireless sensor network localization based on cuckoo search algorithm", Wireless Personal Communications, Vol. 79(1), pp. 223-234, 2014 .

[61] M. Dhivya, M. Sundarambal, J. O. Vincent, "Energy Efficient Cluster Formation in Wireless Sensor Networks using Cuckoo Search", Swarm, Evolutionary and Memetic Computing, pp. 140-147, 2011.

[62] Z. Cui, X. Cai, "Optimal Coverage Configuration with Social Emotional Optimisation Algorithm in Wireless Sensor Networks", International Journal of Wireless and Mobile Computing, Vol. 5(1), pp. 43-47, 2011.

[63] D. C. Hoang, R. Kumar, S. K. Panda, "Optimal Data Aggregation Tree in Wireless Sensor Networks based on Intelligent Water Drops Algorithm", IET Wireless Sensor Systems, Vol. 2(3), pp. 282-292, 2012.

[64] X. Cai, L. Wang, Q. Kang, Q. Wu, "Adaptive Bat Algorithm for Coverage of Wireless Sensor Network", International Journal of Wireless and Mobile Computing, Vol. 8(3), pp. 271-276, 2015.

[65] A. Rajagopal, S. Somasundaram, B. Sowmya, T. Suguna, "Soft Computing Based Cluster Head Selection in Wireless Sensor Network Using Bacterial Foraging Optimization Algorithm", International Journal of Electrical, Computer, Energetic, Electronic and Communication Engineering, Vol. 9(3), pp. 379-384, 2015.

[66] Y. Ding, R. Chen, K. Hao, "A Rule-driven Multi-path Routing algorithm with Dynamic Immune Clustering for Event-Driven Wireless Sensor Networks", Neurocomputing, Vol. 203, pp. 139-149, 2016.

[67] B. Zeng, Y. Dong, "An Improved Harmony Search based Energy-Efficient Routing Algorithm for Wireless Sensor Networks", Applied Soft Computing, Vol. 41, pp. 135-147, 2016.

[68] A. S. Alsaadi, T. C. Wan, A. Munther, "Application of Harmony Search Optimization Algorithm to Improve Connectivity in Wireless Sensor Network with Non-uniform Density", Journal of Information Science and Engineering, Vol. 31(4), pp. 1475-1489, 2015. 
[69] M. Breza, J. A. McCann, "Lessons in Implementing Bio-inspired Algorithms on Wireless Sensor Networks", NASA/ESA Conference on Adaptive Hardware and Systems, pp. 271-276, 2008.

[70] X. S. Yang, S. F. Chien, T. O. Ting, "Bio-inspired Computation in Telecommunications", Morgan Kaufmann, 2015.

[71] Z. W. Geem, J. H. Kim, G. V. Loganathan, "A New Heuristic Optimization Algorithm: Harmony Search", Simulation, Vol. 76, N. 2, pp. 60-68, 2001.

[72] D. Manjarres, I. Landa-Torres, S. Gil-Lopez, J. Del Ser, M. N. Bilbao, S. Salcedo-Sanz, Z. W. Geem, "A Survey on Applications of the Harmony Search Algorithm", Engineering Applications of Artificial Intelligence, Vol. 26, N. 8, pp. 1818-1831, 2013.

[73] A. G. B. Tettamanzi, M. Tomassini, "Soft Computing: Integrating Evolutionary, Neural, and Fuzzy Systems", Springer, 2001.

[74] D. Pelleg, A. W. Moore, "X-means: Extending K-means with Efficient Estimation of the Number of Clusters", International Conference on Machine Learning, Vol. 1, 2000.

[75] D. Sculley, "Web-Scale K-Means Clustering", International Conference on World Wide Web, pp. 1177-1178, 2010.

[76] J. Knowles, L. Thiele, E. Zitzler, "A Tutorial on the Performance Assessment of Stochastic Multiobjective Optimizers", Technical Report 214, Computer Engineering and Networks Laboratory (TIK), ETH Zurich, 2006.

[77] Open Data Euskadi initiative, http://opendata.euskadi.eus, retrieved on October 2015. 